\title{
Collision dynamics of high-speed droplets upon layers of variable thickness
}

\author{
Kuo-Long Pan · Kai-Ren Cheng • \\ Ping-Chung Chou $\cdot$ Ching-Hua Wang
}

Received: 8 September 2007 /Revised: 9 December 2007/ Accepted: 24 February 2008/Published online: 15 March 2008

(c) Springer-Verlag 2008

\begin{abstract}
The collision dynamics between a droplet and a film has been studied with high-impact energy that can be grouped in a dimensionless Weber number, We, as normalized by surface energy. To accomplish this, we have developed a technique based on cutting of a high-speed jet, which can generate a single droplet with speed up to $23 \mathrm{~m} / \mathrm{s}$ and $W e$ on the order of thousands. It was found that the boundaries indicating the occurrence of a central jet and that of a secondary droplet disintegrated from the jet decreased monotonically with increased dimensionless film thickness, $H$, and remained constant when the film thickness was larger than the crater depth. However, the transition designating multiple droplets that are originated from a central jet shows a non-monotonic trend with the variation of $H$, with a minimum $W e$ being at $H \approx 3$, which is about the maximum crater depth, owing to a tuning behavior. The critical We for splashing that occurs at an early phase immediately after the impact is relatively sensitive to the film thickness only when $H$ is between 1 and 2 , which increases with reduced $H$. At large $W e$ ( $\gtrsim 2,570$ for high $H$ ), the ejected crown is closed to form a bubble and the transition boundary reveals a similar dependence on $H$ as that for creation of a central jet.
\end{abstract}

\section{Introduction}

The collision dynamics of a droplet upon a surface that is either dry or wet plays a crucial role in various disciplines

K.-L. Pan $(\bowtie) \cdot$ K.-R. Cheng · P.-C. Chou · C.-H. Wang

Department of Mechanical Engineering,

National Taiwan University, Taipei, Taiwan, ROC

e-mail: panpeter@ntu.edu.tw of nature and practical interests. For example, meteorological phenomena such as the interaction of rain drops with the earth surface of ocean or soil, meteorite impaction, spray combustion in liquid-fueled combustors, insecticide spraying, ink-jet printing technologies, spray painting and coating, etc. A lot of efforts have been made to comprehend the underlying mechanism of a droplet impinging a solid surface (Stow and Hadfield 1981; Chandra and Avedisian 1991; Yarin and Weiss 1995; Cossali et al. 2005) or a pool that is covered by the same liquid of sufficiently large depth so as to neglect the surface effect (Jayaratne and Mason 1964; Engel 1966; Rodriguez and Mesler 1985). It is in the near decade that more attention was paid to the collision between a droplet and a liquid layer with finite thickness, both experimentally (Rioboo et al. 2003; Okawa et al. 2006) and theoretically (Roisman and Tropea 2002). An overview of the problem can be referred to a recent review of Yarin (2006).

Considering head-on collision of a droplet upon a layer of the same liquid with small impact inertia, it is expectable that the slowly approaching interfaces would eventually coalesce, due to the attraction of intermolecular force. As the impact energy or liquid deformability is increased, more gas will be trapped within the gap between the deformed surfaces. Consequently, higher pressure is built up and resists further approaching of the droplet; it thus results in a bouncing regime. Here a dominant dimensionless parameter for characterizing a regime diagram is the Weber number which identifies the ratio of impact inertia to surface force (Jiang et al. 1992; Qian and Law 1997; Pan and Law 2007), We $=2 \rho_{\ell} V_{r e l}^{2} R / \sigma$, where $V_{\text {rel }}$ is the relative velocity, $R$ the radius of the droplet, and $\rho_{\ell}$ and $\sigma$, respectively, the density and surface tension of the liquid. If $W e$ is further increased, however, the repulsive pressure inside the gap is overwhelmed by stronger impact 
inertia and the surfaces will merge again. With even higher $W e$, the merged liquid has to separate in order to balance the excess energy that cannot be digested in a single layer merely by ensuing deformation or viscous loss during the oscillation. Depending on the relative magnitudes of impact inertia, surface tension, and viscous dissipation, a myriad of secondary droplets can be further created; a typical phenomenon is known as splashing.

Previous studies were mostly focused on the characteristics of splashing and its transition mechanisms beyond the regime of total absorption, which occurs when the impaction is sufficiently harsh (Worthington 1908; Hobbs and Kezweeny 1967; Rodriguez and Mesler 1985; Vander Wal et al. 2006). In that situation, a crown is formed and multitude of secondary droplets are further disintegrated from the rim after a corrugated shape is generated by the capillary instability, which is then followed by the ejection of a central column known as Rayleigh or Worthington jet (Shin and McMahon 1990). To further understand the interaction between a droplet and surface, we have investigated the regimes of bouncing and absorption that occur at lower We (Pan and Law 2007). In this work, however, we aim to study the regimes at higher $W e$ 's, which go beyond the general range, say, larger than 1,000. Furthermore, the effect of varied thickness of the liquid layer, when it is of finite value that is close to the droplet diameter, is emphasized. While some researchers have investigated the influence of finite thickness on the splashing, mostly concerning the height of the Worthington jet or the number of droplets released from the jet (Hobbs and Osheroff 1967; Shin and McMahon 1990), or the maximum radius of the crater, as was considered by Macklin and Metaxas (1976), in this study, we have systematically investigated the transition boundaries as We is increased from $O(10)$ to thousands, to catch the characteristics of different modes that may occur beyond the nominal splashing and their variation with the depth of the layer.

To achieve the test condition of high-speed impact, we have developed a droplet-generating method that is different from conventional approaches that adopt methods such as vibration of piezoelectric diaphragms (Ashgriz and Poo 1990; Jiang et al. 1992) or creation of multiple droplets due to Rayleigh instability either via wave modulation (Jayaratne and Mason 1964; Adam et al. 1968) or naturally during free dripping (Hobbs and Kezweeny 1967; Rodriguez and Mesler 1985), by which the droplet speed is generally on the order of $1 \mathrm{~m} / \mathrm{s}$. By means of the present cutting technique via a high-speed water jet, we can create moderately stable droplets associated with velocity nominally as high as $23 \mathrm{~m} / \mathrm{s}$, hence leading to a Weber number up to 5,100 . With such strong impact, the envelope of the ejected sheet at the rim was observed to close, which further generated two jets that extended, respectively, upward and downward from the dome and were distinct from the Worthington jet. This technique, nevertheless, encounters difficulties that impede further increase of Weber number, due to inherent oscillation of the cut droplets and aerodynamic deceleration of viscosity of surrounding fluid. The scenario observed herein provides also a reference for those adopting electronic valves for controlling breaking of a liquid jet.

\section{Experimental method and setup}

\subsection{Apparatus for generating high-speed droplets}

A water jet with adjustable speed was generated by the thrust of a gas cylinder driven by a compressor, which was equipped with a movable piston linked to two liquid cylinders, as sketched in Fig. 1. The measurement of the movement was conducted by Mitutoyo's DRO (Digital Readout) that integrated the AT100 Series Linear Scales with the K Series dedicated digital counters, which offered accurate detection and display of axial displacements of the pistons (up to $0.001 \mathrm{~mm}$ ) that were used as indications of the flow rate and hence, jet speed. Due to compression, the liquid within two smaller cylinders downstream was injected out. The jet, passing through filters and eventually squeezed out of a nozzle with given exit diameter of $0.45 \mathrm{~mm}$, was continuously discharged into a chamber (Fig. 1) that was made of Plexiglas walls and used to isolate the influence of outside air. Depending on the upstream pressure controlled by a valve, the speed of the jet was able to go beyond $50 \mathrm{~m} / \mathrm{s}$ (the full scale of pressurization was $10 \mathrm{~atm}$ gauge pressure while $3 \mathrm{~atm}$ was about to accelerate the liquid to $50 \mathrm{~m} / \mathrm{s}$ at the exit of the nozzle).

The diameter of the jet did not change much, which was about the size of the nozzle, before impinging a rotating disk at a distance of $4 \mathrm{~cm}$. With high-speed rotation of the disk that was mounted with a knife-edge and driven by a motor, the jet was cut in pieces, which subsequently formed droplets. Figure 2 shows that, if the rotation is too fast $(2,900 \mathrm{rpm})$, the cut portion of the jet is so short (about four times of the jet radius, $a$ ) that the remaining mass cannot cohere well by the surface tension and tends to disintegrate as distributed droplets due to large perturbation of the cutting, as marked by a rectangle in the figure. The following small droplets were generated by the entailed film that was caused by the liquid accumulated on the disk due to continuous impingement of the jet. If the rotating speed is too low $(630 \mathrm{rpm})$, however, substantial portion of the jet is cut off (Fig. 3a), leading to disintegration of multiple droplets. The breakup mechanism would be relevant to the Rayleigh instability (Rayleigh 1879; Eggers 
Fig. 1 Schematic diagram of the experiment setup

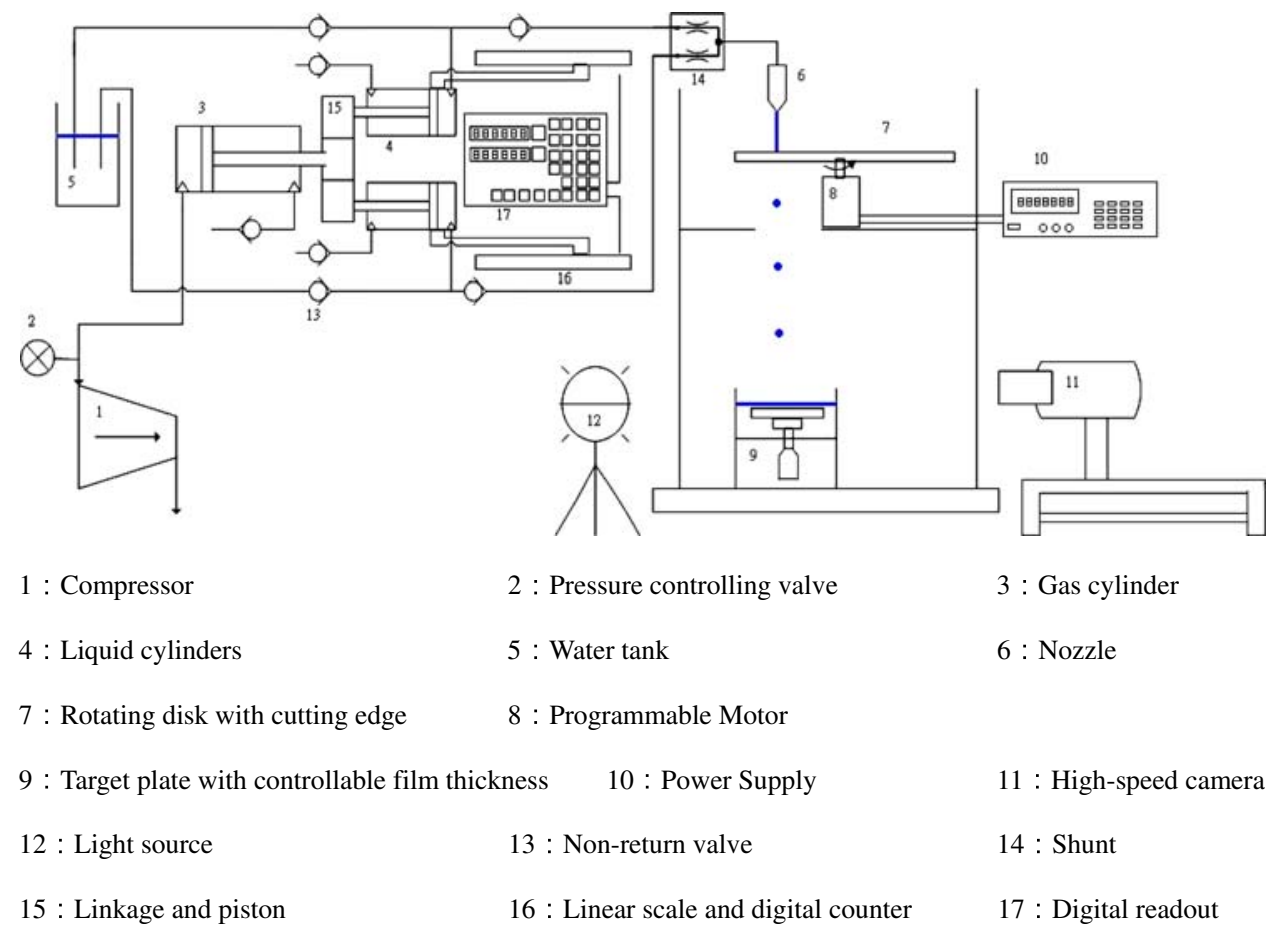

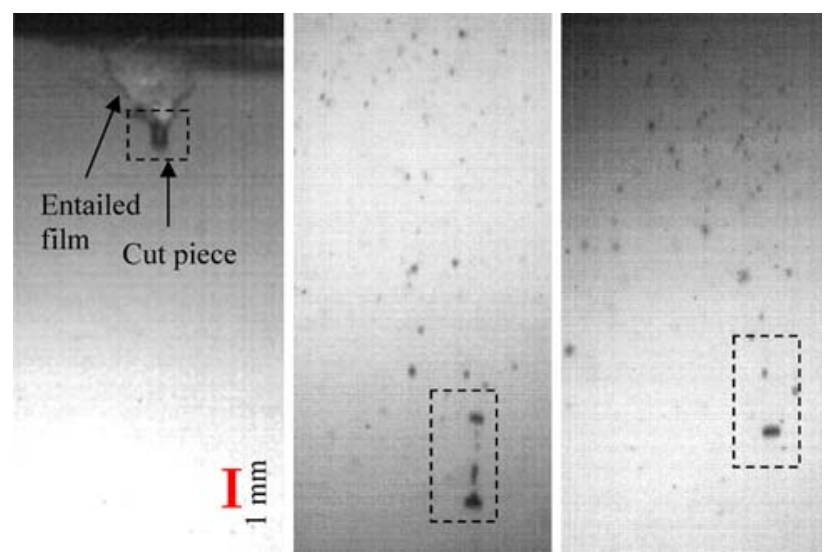

Fig. 2 The pictures of a jet cut by a disk rotating at 2,900 rpm, captured by a high-speed camera at the locations immediately below the disk (left column), $1.5 \mathrm{~cm}$ lower (central column), and $3.0 \mathrm{~cm}$ lower (right column). The cut length is about $4 a$. The mass cut by the disk is enclosed by a rectangle and a length bar is included to indicate the scale of $1 \mathrm{~mm}$

1997) that exerts on the long liquid thread. However, a recent study (Marmottant and Villermaux 2004) shows that stretching effect will yield interaction of liquid blobs in the ligament and affect the distribution of droplet size after pinch off such that it is not as uniform as predicted by the Rayleigh instability. While the droplets created by the present cutting technique are indeed not uniform, we reserve our conclusion on the definite mechanism leading to breakup of the droplets, which shall need systematic investigation in terms of well-controlled tangential forces that are driven by sources different from the normal stretching of Marmottant and Villermaux (2004) and discharged jet possibly at low speed. If the speed of rotation is increased $(1,833 \mathrm{rpm})$, the cut amount is reduced and thus a single piece that carries most of the original liquid can be formed, as demonstrated in Fig. 3b. Due to oscillation of the deformable surface, however, the droplet is not fixed in spherical shape within the given distance of traveling; the average fluctuation of the axes is $5.97 \%$ with an aspect ratio of width to length being 1.48 . If the rotating speed of the disk is further increased $(2,167 \mathrm{rpm})$, the size of the formed droplet is reduced and consequently the oscillation can be more quickly relaxed, yielding a spherical droplet before the target of collision is reached (Fig. 3c) with which the fluctuation error is $0.94 \%$ and the aspect ratio is 0.97 . Intriguingly, the cut length is $8.7 a$, which is about the value that exhibits the maximum rate for the Rayleigh instability to grow, i.e. $9 a$, and hence is possibly the length to be cut in the easiest manner to form a single droplet.

It is noted that, while secondary droplets of smaller size might be generated immediately after the head of the jet was cut off, they could not catch up with the major element because relatively larger viscous air drag substantially decelerated their following motions. Therefore, they did not affect the collision of the main droplet with the target surface, as long as the first one was captured. We further note that, the speed of the fastest droplet that became nearly spherical and stable was $23 \mathrm{~m} / \mathrm{s}$. It was generated 
Fig. 3 The transient images of droplets at the locations immediately below the disk $(3 \mathrm{~cm})$, a little downstream $(6 \mathrm{~cm})$, in the middle $(60 \mathrm{~cm})$, and before the target plate $(120 \mathrm{~cm})$, respectively shown from the top panel to the bottom panel. The rotation speed of the disk and the cut length are: a $630 \mathrm{rpm}, 40 a$; b $1,833 \mathrm{rpm}$, $12 a$; c $2,167 \mathrm{rpm}, 8.7 a$. A length bar is included to indicate the scale of $1 \mathrm{~mm}$
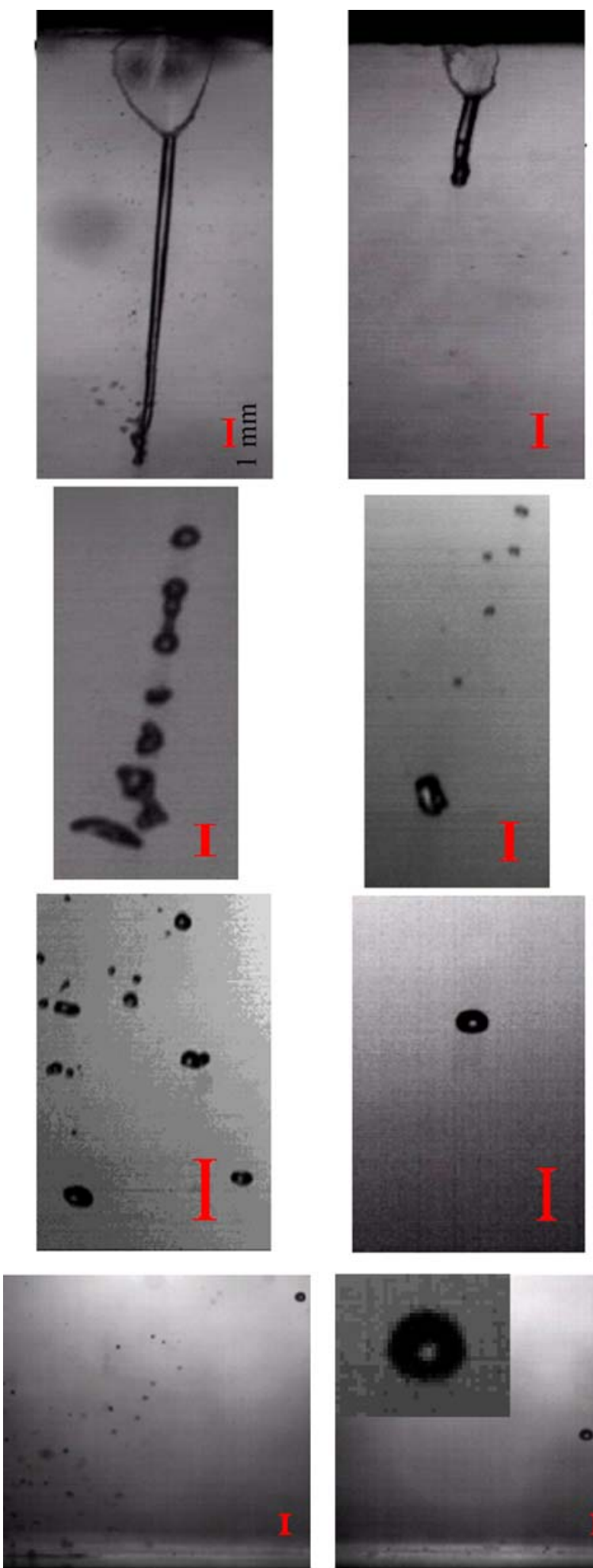

(a)
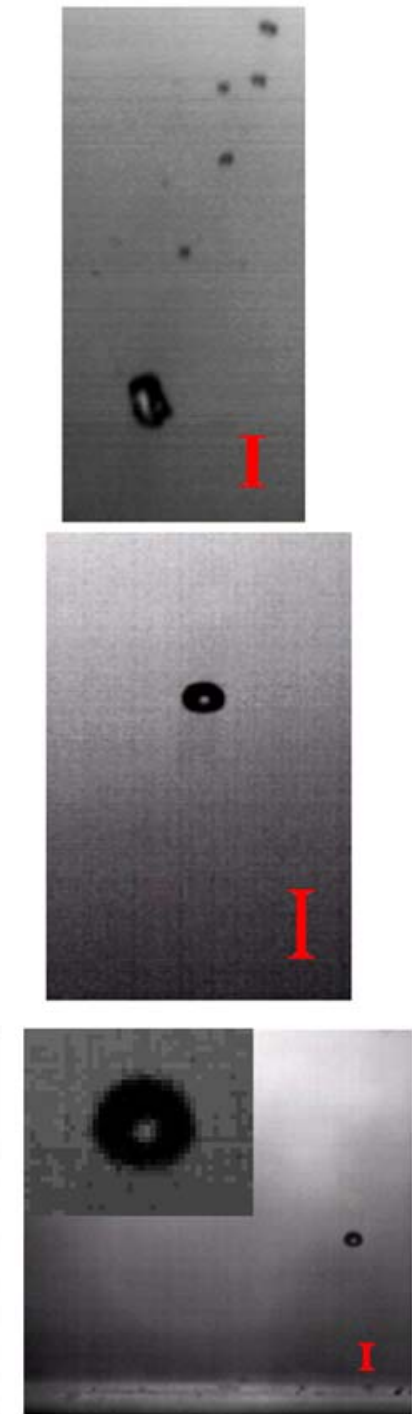

(b)
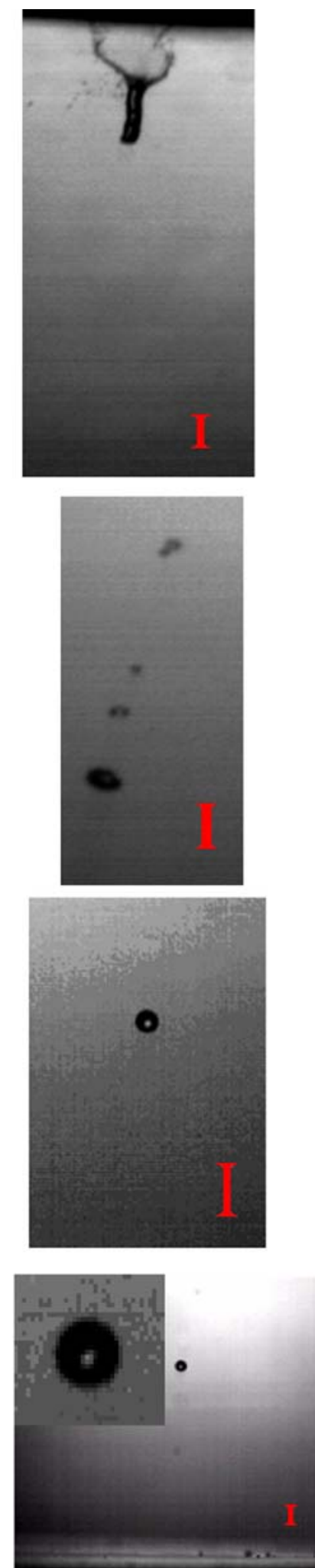

(c) initially with a speed, which was close to that of discharged jet, after the cutting of the rotating disk, but was decelerated rapidly by the viscous air drag, as demonstrated by the velocity variation with respect to the distance, $Z$, shown in Fig. 4. This inherently limits the range of attainable Weber number for a stable, spherical droplet that shall enable us to 


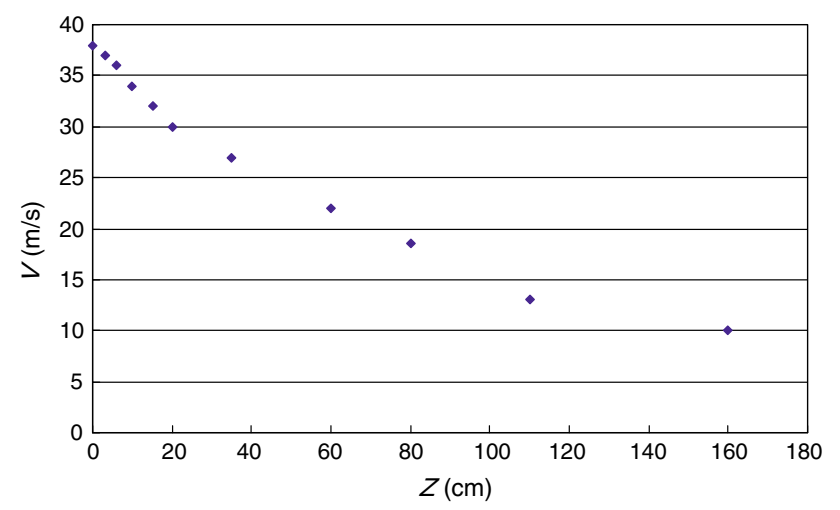

Fig. 4 The variation of droplet velocity $(V)$ with respect to the distance measured from the rotating disk $(Z)$ for the case of Fig. 3c, in which the collision was made at $Z=60 \mathrm{~cm}$ whereby satisfactory sphericity and stability had been obtained

quantitatively assess the transition boundaries, but not in an oscillating manner (Xu et al. 2005). This difficulty would be overcome by a near vacuum condition but then liquids with low vapor pressure should be adopted to avoid evaporation (Orme and Muntz 1987).

\subsection{Test section and data acquisition}

Considering the higher stability and easier control of droplet generation, the range of lower Weber number (We $\lesssim 2,000$ ) was investigated with free falling of droplets that dripped from a syringe or nozzle, with the velocity being adjusted by the height. Three types of surfaces have been tested, i.e. a dry surface, a pool, and a film, with thickness on the order of the impinging droplet size. To control the thickness of the film, a circular plate was immersed in a tank with fixed level of water (Fig. 1). By adjusting the height of the plate via the procession of a manipulating micrometer at the bottom which is equipped with a cylindrical Vernier scale, the distance between the surfaces of the solid plate and the immersion liquid can be controlled to as fine as $200 \mu \mathrm{m}$. The minimum thickness that can be attained is limited by the strong surface tension of water, which yields a large contact angle between the surfaces of liquid and solid, specifically around the corner and thus prohibits the film from being uniformly spread on the solid surface when the layer is thin (de Gennes 1985). To avoid oscillation of droplets while maintaining sufficiently large $W e$, which worsens with increased droplet size, the diameter of droplets using the cutting methodology was kept below $1 \mathrm{~mm}$, typically about $700 \mu \mathrm{m}$ or smaller, and hence the minimum dimensionless thickness of the film $(H \equiv h / D$, the physical thickness normalized by the droplet diameter) was about 0.3. The solid surface tested was a polished smooth Plexiglas plate.

The visual observation was recorded by a high-speed CMOS digital camera (X-Stream ${ }^{\mathrm{TM}}$ Vision, XS-4), which supported a maximum resolution of $512 \times 512$ pixels with 5,100 frames per second (fps). Depending on the test condition, the frame rate can be raised to $20,000 \mathrm{fps}$, while the resolution is shrunk to $128 \times 512$. The images at low $W e$ were captured with satisfactory quality mostly based on $10 \mu$ s exposure time and continuous illuminating. For large $W e$, however, the shutter of the camera was synchronized with a LED lamp that can support the shortest duration of $1 \mu \mathrm{s}$, to capture images with sufficiently small exposure and adequate light intensity while avoiding slurring due to background scattering.

In passing, we note that another parameter of importance, which is not reported herein, is the liquid viscosity since it may affect the extent of energy dissipation due to the liquid motion. Its effect can be presented in terms of variation of Reynolds number or Ohnesorge number and shall be systematically studied using liquids of different viscosities. With constant property of water in this work, we emphasize the variation of Weber number that has been well reported to characterize the transition of collision outcome with different impact inertia and surface deformation (Ashgriz and Poo 1990; Jiang et al. 1992; Qian and Law 1997; Cossali et al. 2004; Pan and Law 2007) and should suffice to indicate the critical behaviors of transition boundaries with respect to $H$.

\section{Results and discussion}

\subsection{Regime diagram}

In addition to a film, a dry surface and a pool have also been adopted as the collision target so that the critical boundaries can be quantitatively compared. When We is increased, the outcome of a droplet impacting a dry surface changes from "spreading" of a flattened disk (Fig. 5a), to "fingering" of the circumference (Fig. 5b), and to "breakup" of the fingers (Fig. 5c), yielding multiple droplets shed from the rim. The mechanisms have been well known to the community (Rein 1993; Xu et al. 2005). If a layer of the same liquid is added on the surface, however, the outcome becomes richer. Specifically, as We is increased from the order of 10 , the droplet merges with the layer, followed by appearance of a central jet after merging (Fig. 5d), which is accompanied by breaking of the neck and hence, a secondary droplet when $W e$ becomes larger (Fig. 5e); more droplets are created if We is further increased (Fig. 5f). A sheet is formed at the rim if We is large enough, leading to a precursor of splashing of multiple droplets (Fig. $5 \mathrm{~g}$ ) after the rim is wrinkled at high We due to the rim transverse instability (Roisman et al. 2006; Bagué et al. 2007). It is noted that, before merging can be observed as We is sufficiently large, bouncing may occur 
Fig. 5 The typical feature of a droplet impacting a dry surface (top view): a spreading; b fingering; c breakup, and a droplet impacting a wet surface (side view): $\mathbf{d}$ central jet; e central jet followed by a secondary droplet; $\mathbf{f}$ central jet followed by multiple droplets; g splashing

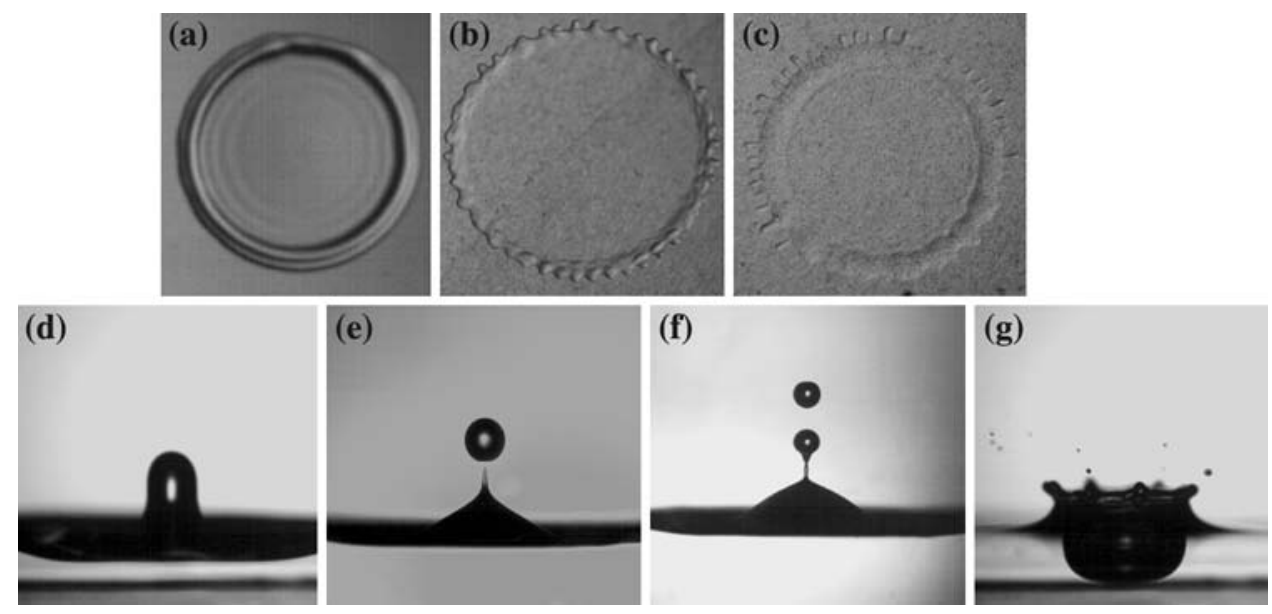

due to relatively larger repulsive pressure inside the gas gap between the interfaces than the impact inertia, which impedes contact of the droplet with the surface of the liquid layer (Couder et al. 2005; Pan and Law 2007). In our study, since it happens at pretty low We that has not been systematically identified using current apparatus, the transition is not marked on the regime diagram. If $W e$ is further decreased, however, it is expected that another regime of merging shall appear, whereas absorption of the droplet by the surface is dominated by the intermolecular attraction, while the significance of hydrodynamics is negligible (Pan and Law 2004).

The colliding scenarios associated with moderately large impact inertia have been well reported (Worthington 1908; Rein 1993), while herein we purposely identify the transitional boundaries in terms of $W e$, specifically for characteristic modes as transforming from absorption to splashing and beyond, with respect to the variation of film thickness, which is elusive in the literature but shall facilitate the analysis of the hydrodynamic behavior. Figure 6 shows the diagram of different regimes in terms of the Weber number versus the dimensionless thickness of the layer, $H$. It is seen that, when $W e$ is increased, the boundaries demarcate regimes of absorption of the droplet by the film (regime A), appearance of a central jet (regime C), a central jet followed by a secondary droplet pinched from the jet (regime S), and a central jet followed by multiple droplets pinched from the jet (regime $\mathrm{M}$ ). In addition, a boundary marked by a "splashing" curve intercepts these regimes, indicating breakup of the fingers growing at the rim of ejected sheet and splattering of multiple droplets from the rim. At higher We, splashing and a central jet entailed with multiple droplets was observed and the characteristic phenomena kept invariant until $W e \approx 2,570$.

An interesting regime when We becomes higher is closure of the ejected crown, as shown in Fig. 7. It is seen that the sheet at the rim is bent inward and then encloses the cavity formed by initial crater, leading to a large bubble with jets extended both upward and downward from the dome. The downward jet is then pulled back by surface tension or may further interact with the Worthington jet that later comes out from the bottom film, which however is not shown in this condition. The upward jet is subsequently pinched off as it extends so that a secondary droplet is formed, while its origin of creation is different from that of the Worthington jet. With increase of We,

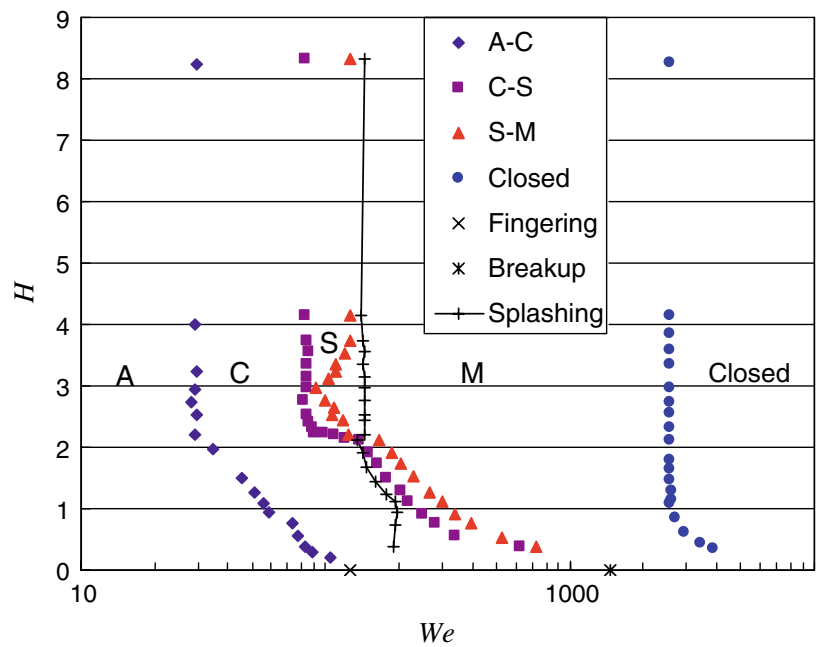

Fig. 6 The regime diagram of droplet collision on a wet/dry surface in terms of $W e$ versus $H$. The boundary $A-C$ indicates the transition from absorption (regime $A$ ) to appearance of a central jet (regime $C$ ); $C-S$ is the boundary between regime $C$ and that followed by a second droplet (regime $S$ ); $S-M$ divides regime $S$ and that associated with multiple droplets pinched from a central jet (regime $M$ ). The curve "splashing" designates the occurrence of multiple droplets shed from the sheet of the ejected crown. The crown is wrapped up as We is larger than that marked by "closed". The collision of a droplet on a dry surface transforms from spreading, to formation of fingers, and to breaking of the fingers into multiple droplets, as divided by the symbols on $H=0$ axis 
Fig. 7 The collision sequence demonstrates closure of the ejected crown for $W e=3,953$ and $H=2.1(V=20.02 \mathrm{~m} / \mathrm{s}$, $D=0.71 \mathrm{~mm}, h=1.5 \mathrm{~mm}$ ); $\Delta t=0.8 \mathrm{~ms}$
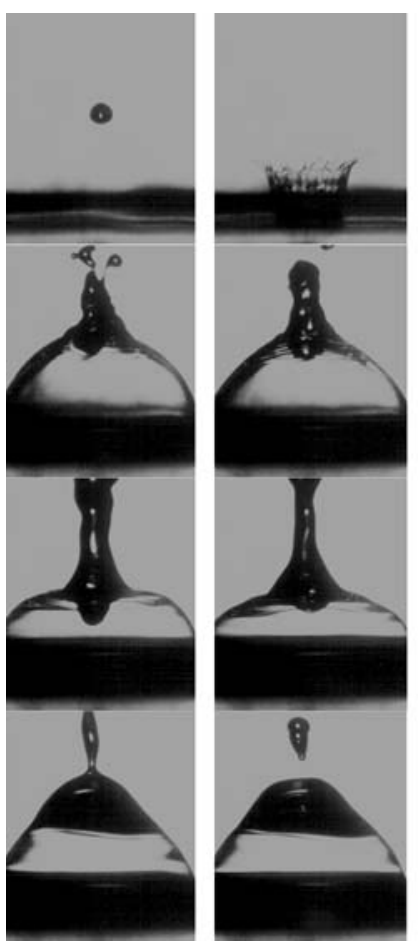
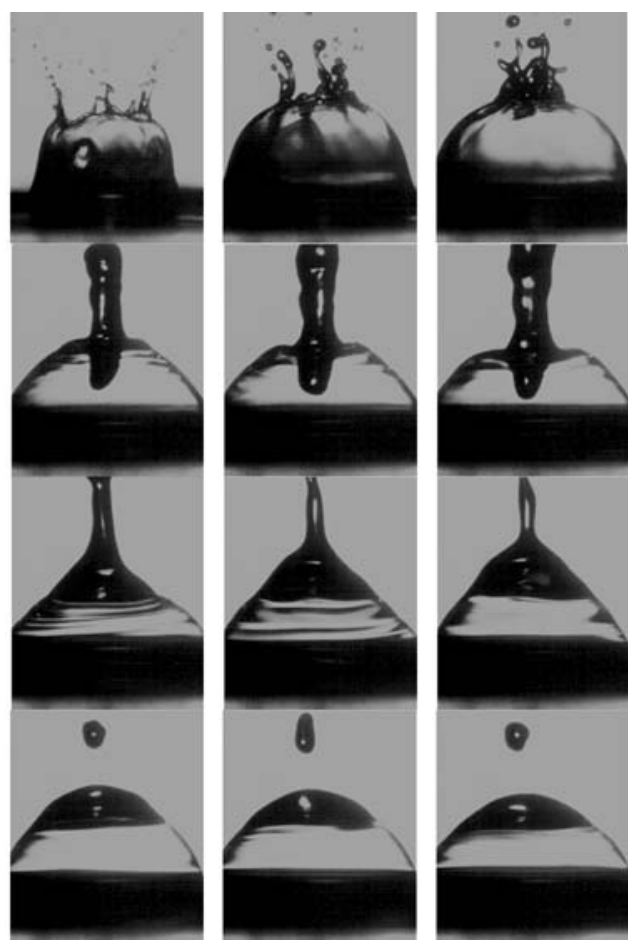

more droplets will be emitted from the jet, analogous to the scenario of multiple droplets yielded by the central jet in regimes $\mathrm{S}$ and $\mathrm{M}$. Although similar phenomena have been observed previously by Worthington (1908) and rephrased by Engel (1966), the difference between a film and a pool has not been elucidated clearly as far as the authors know. Meanwhile, a quantitative study of the critical Weber number as well as its variation with the dimensionless thickness of the film should be first reported herein, as discussed in the following.

\subsection{Effect of film thickness on the transition of collision outcome}

The regime diagram (Fig. 6) indicates that, as the dimensionless film thickness is increased, the Weber numbers of the characteristic boundaries that occur beyond absorption decrease monotonically, specifically as $H \lesssim 1$, and approach constant as $H \gtrsim 2$. These boundaries identify the various scenarios to be created after the impact droplet is absorbed by the liquid layer. To investigate the mechanism leading to the variations, we have classified the boundaries based on the changes due to the central jet and that due to the ejected sheet at the rim, which occur at different phases of the collision. As We is large enough, a Worthington jet is ejected by the recirculated flow that is thrust outward initially by the impact. A sheet at the rim is formed beforehand if $W e$ is further increased. These phenomena are categorized into regime $\mathrm{C}$ as marked in Fig. 6 . The variation of the boundary that divides regime $\mathrm{A}$, where absorption dominates, and regime $\mathrm{C}$ with respect to $H$ can be understood via the dependence of flow structure on the depth of liquid. If the film is thin $(H=0.38)$, as shown in Fig. 8, the impacted crater quickly encounters the bottom, i.e. the solid surface. Consequently the forwarding motion is retarded so that the flow coming back to refill the cavity from the surrounding layer of small thickness does not carry inertia and energy large enough to generate a magnificent central jet, as contrasted by Fig. 9 for a thick film $(H=3.85)$. Therefore the transition We increases with lowered $H$.

The mechanism to create a secondary droplet out of the central jet similarly relies on the kinetic energy of the retrieving flow motion to form a jet. Thus the boundary separating regime $\mathrm{C}$ and regime $\mathrm{S}$ has a similar trend as that indicates appearance of a central jet. To generate more droplets from the jet, however, a non-monotonic variation of the boundary was observed as $2 \lesssim H \lesssim 4$ with a turning point at $H \approx 3$. This can be realized in terms of the tuning phenomenon (Hobbs and Osheroff 1967; Shin and McMahon 1990) via which the jet is associated with largest height and pinched off with most droplets when the film thickness is about the maximum depth of the crater. The reason was, as argued by Macklin and Hobbs (1969), that the upward motion of the jet was rendered largest momentum due to the change of pressure gradient at the bottom of the crater from the horizontal to vertical direction. It is seen in Fig. 10 that a much thinner jet, later disintegrated into several pieces, is ejected after the crater touches the solid surface and a pin is formed at the bottom 
Fig. 8 The collision sequence of a droplet with $W e=124$ and $H=0.38(V=1.853 \mathrm{~m} / \mathrm{s}$, $D=2.6 \mathrm{~mm}, h=1.0 \mathrm{~mm})$; $\Delta t=1.8 \mathrm{~ms}$
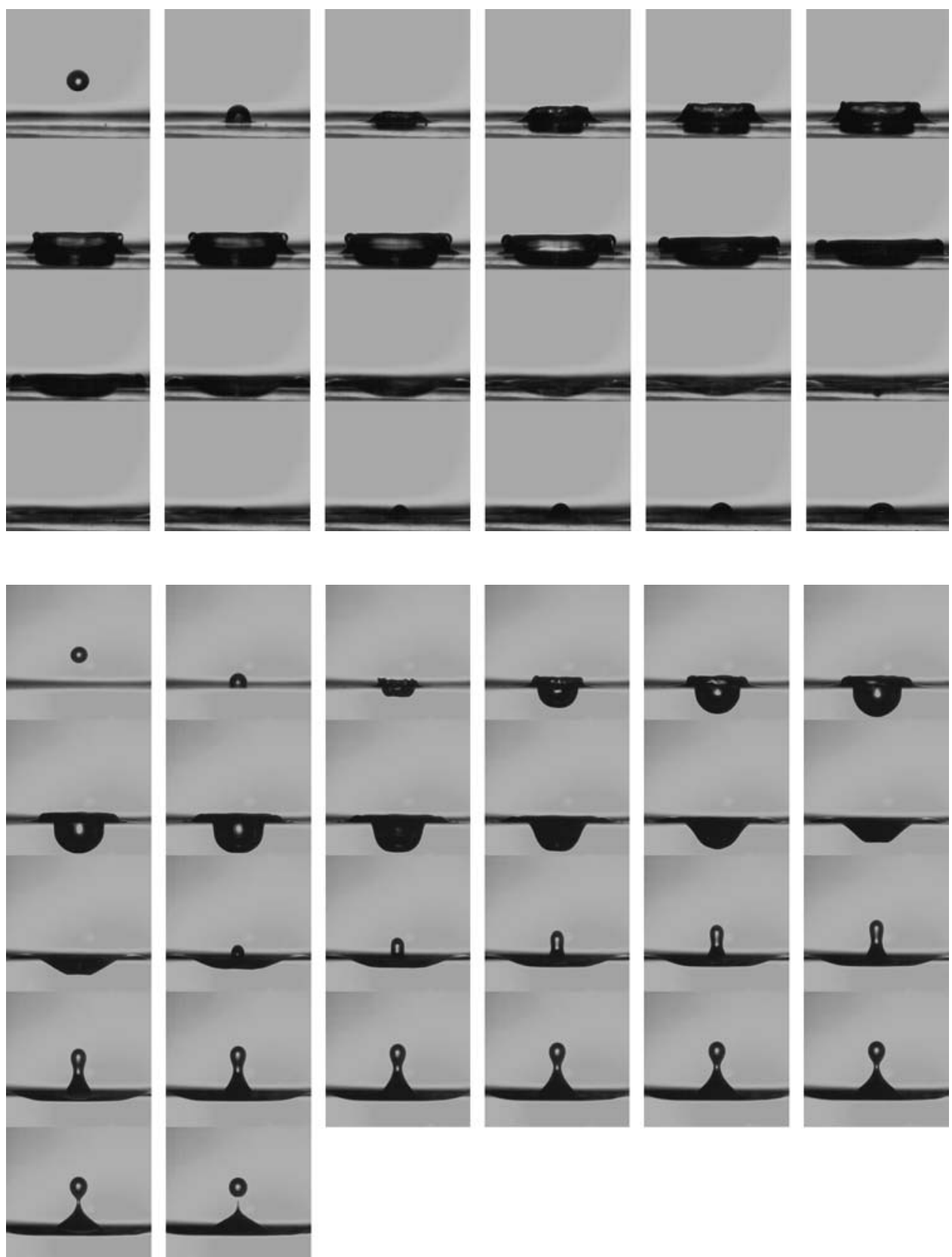

Fig. 9 The collision sequence $H=3.85(V=1.853 \mathrm{~m} / \mathrm{s}$,
$D=2.6 \mathrm{~m}$, $D=2.6 \mathrm{~mm}, h=10 \mathrm{~mm})$; $\Delta t=1.8 \mathrm{~ms}$

$(H=2.5)$ that is later rebounded upward with substantial energy due to surface tension. This is distinguished clearly from the thick jet and smoother bottom surface of the crater as shown in Fig. 9 when the film thickness is larger than the depth of the cavity.

These regimes are intercepted by a curve, designating the transition of a fingering sheet to formation of multiple droplets ejected from the rim. This phenomenon typically occurs much earlier than the central jet and is generally yielded by sufficiently large impact energy. When the film thickness is smaller, however, the transformation becomes more difficult, as shown in Fig. 11a where a slightly wrinkled sheet is formed at beginning of the impact. If $H$ is increased so as to cross the transition boundary, multiple droplets are shed from the rim of the sheet, leading to splashing (Fig. 11b), which is followed by a thinner jet that is associated with larger kinetic energy upward as discussed. Since this happens before the crater is fully developed and encounters the solid surface, it reveals that the depth of the layer, specifically as it is of the same order as the droplet size, could have affected other factors such as the capillary instability or an ejecta sheet from the film (Thoroddsen 2002) that might trigger a precursor of splashing while the retrieving flow is still far from being effective in changing the upward motion. This effect, nevertheless, seems to saturate when $H$ is smaller than 1 
Fig. 10 The collision sequence of a droplet with $W e=124$ and $H=2.5(V=1.853 \mathrm{~m} / \mathrm{s}$, $D=2.6 \mathrm{~mm}, h=6.5 \mathrm{~mm})$; $\Delta t=1.8 \mathrm{~ms}$
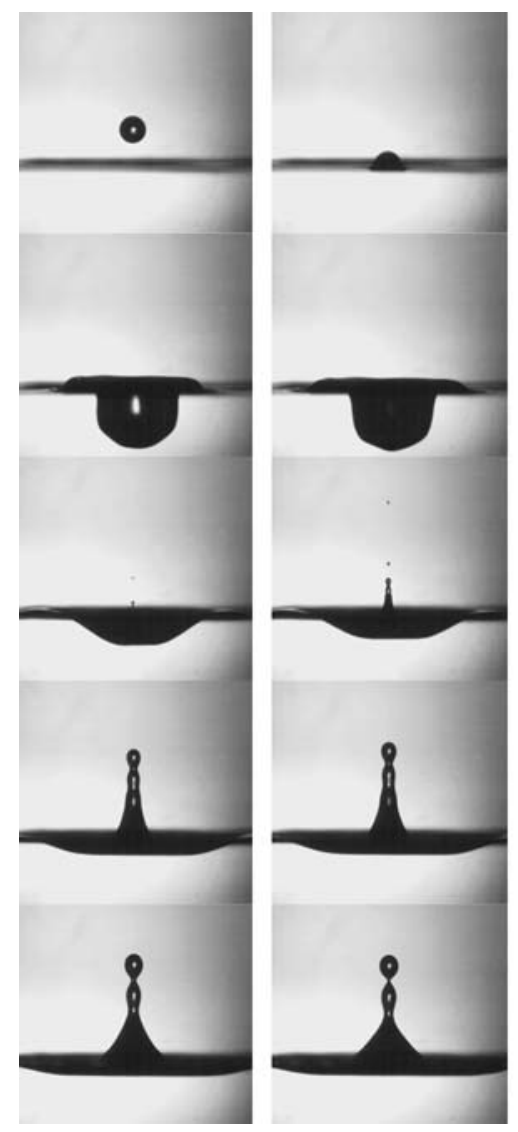
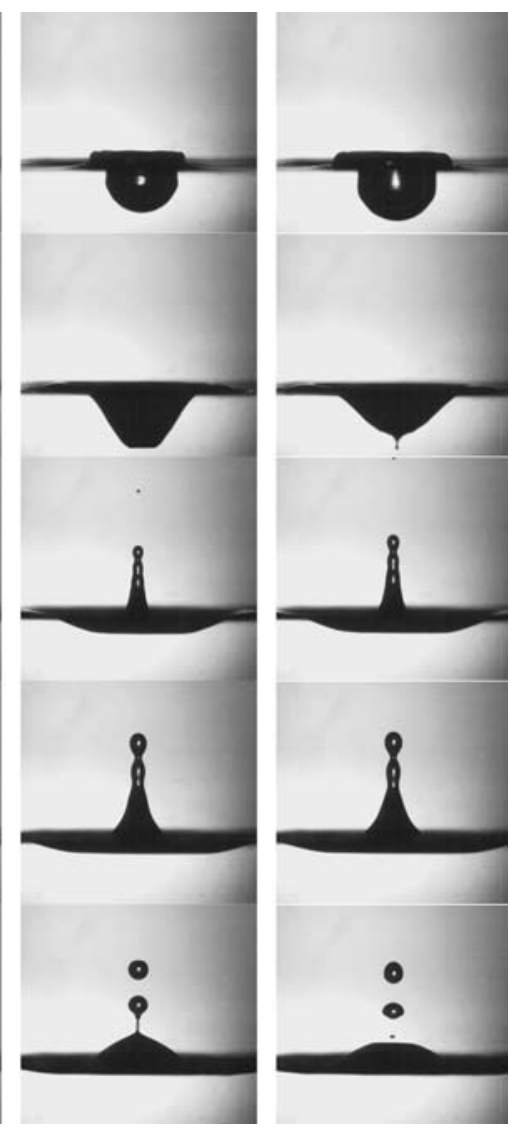

whereby the boundary is almost invariant with respect to $H$. We note that in the work of Cossali et al. (1997), the critical Weber number of water in fact did not rise noticeably with increasing $H$ as other fluids used in their study as they stated but was appearing nearly constant when $H \gtrsim 0.2$. Furthermore, according to the small variation below $H \approx 0.2$ shown in their plot and the results of very thin film $(H \lesssim 0.1)$ of Wang and Chen (2000), we conjecture that this boundary for water is somewhat insensitive to the film thickness when it is smaller than the droplet diameter. More detailed analyses that integrate theory and numerical simulation would help explore the governing mechanism.

The transition boundary for the crown to wrap up occurs at $W e \approx 2,570$ for sufficiently large film thickness but increases with decreasing thickness as $H \lesssim 1$. The reason can be inferred from the evolution pictures for different layer depths, as shown in Fig. 12. It is seen that if $H$ is large (Fig. 12a), the sheet ejected from the rim is strong enough to reach the dome and close the crown. Since it is at the margin of occurrence, the closed cavity is soon collapsed by the pull of gravity. The bubble inside is then pushed downward while it rises later to the water surface due to buoyancy. In a thin film (Fig. 12b), however, the ejection of the sheet in the beginning is weak and so the rim bent inward tumbles before it converges to the central peak. Accordingly, the factor for the crown to close relies on the balance between the upward motion of the sheet and the downward pull of gravity. It is thus expectable that the tendency to enclose the crown would be reduced with lowered film depth whereby the ejection inertia becomes weaker, analogous to that for creations of a central jet and that further entailed with a secondary droplet, thereby leading to increasing Weber number for observation.

It is noted that the transition Weber numbers for fingering and breakup on a dry surface are, respectively, close to the extensions of transition boundaries indicating appearances of a central jet $(\mathrm{A}-\mathrm{C})$ and multiple droplets (S-M) on a wet surface. It may imply certain connections between these structures while we have not been able to claim yet.

\section{Concluding remarks}

A technique for generation of high-speed droplets, nominally up to $23 \mathrm{~m} / \mathrm{s}$, has been preliminarily developed and tested. Different from conventional approaches in terms of vibration of piezoelectric diaphragms or creation of multiple droplets due to the Rayleigh instability either via 
Fig. 11 The collision sequence of a droplet with $W e=176$ $(V=2.208 \mathrm{~m} / \mathrm{s}, D=2.6 \mathrm{~mm})$ for $\mathbf{a} H=1.15$ and $\mathbf{b} H=1.35$; $\Delta t=2.1 \mathrm{~ms}$
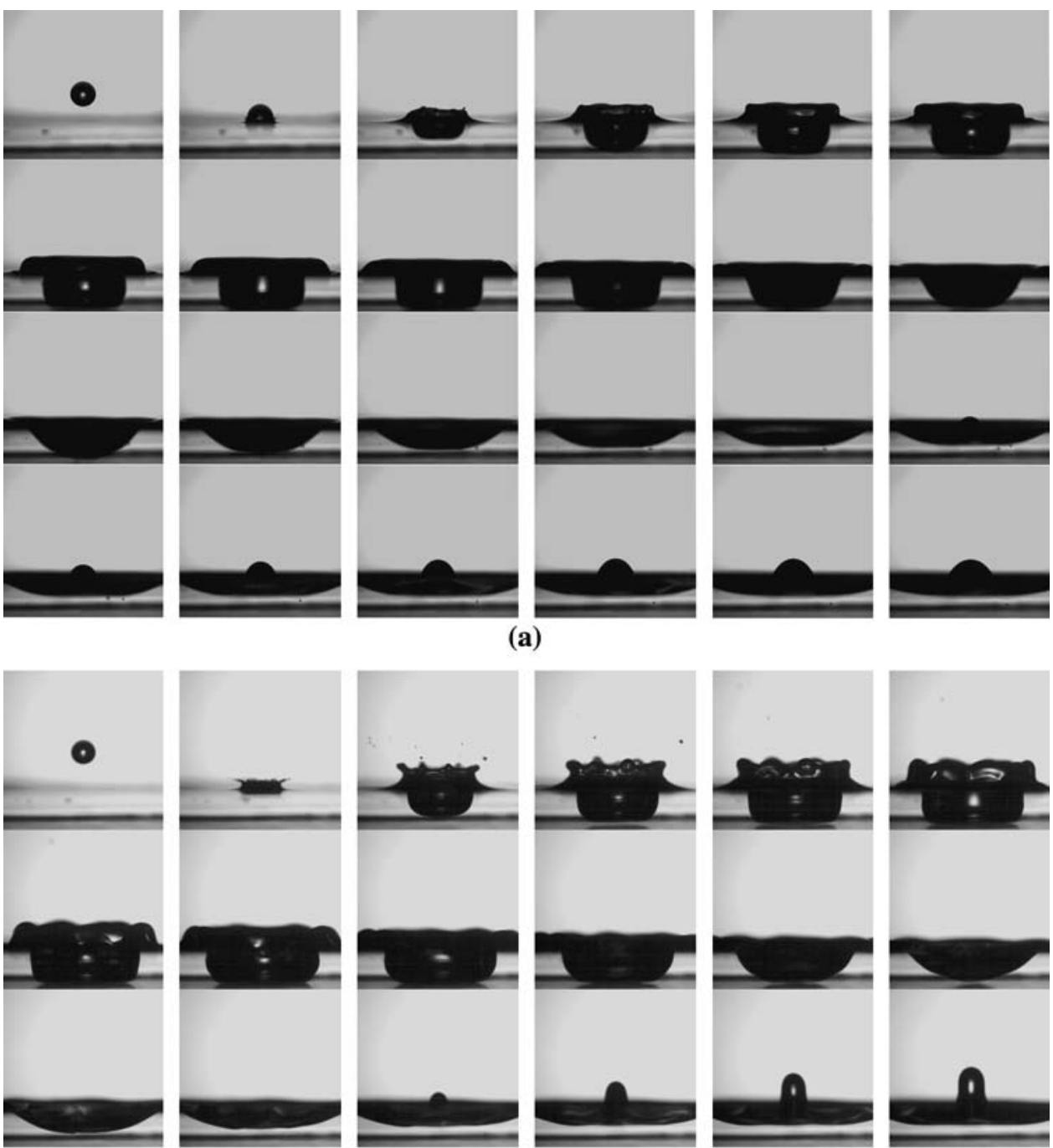
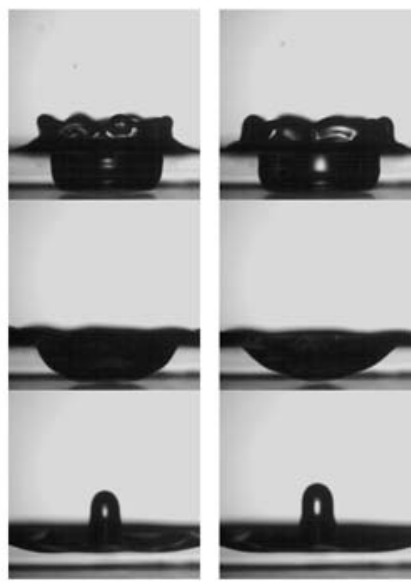
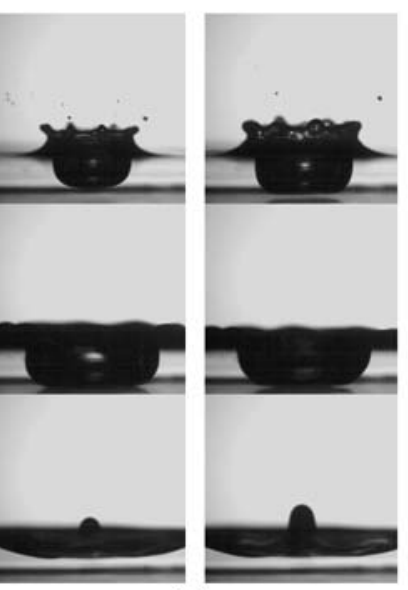

(b) .

wave modulation or naturally during free dripping, by which the produced droplet speed is typically on the order of $1 \mathrm{~m} / \mathrm{s}$, the methodology is based on the cutting of a highspeed jet, which can be easily generated by the procession of a piston equipped on a gas cylinder. While the jet speed can be accelerated to higher than $50 \mathrm{~m} / \mathrm{s}$, the maximum Weber number is limited by the formation of spherical droplets dominated by the oscillation that is manifested with large droplets $(D \cong 1 \mathrm{~mm})$, and the drag force that exerts larger retardation for smaller droplets. So far the highest Weber number we have obtained for a moderately stable droplet is about 5,100, which was yielded by a cut length of the jet close to that associated with maximum growth rate of Rayleigh instability.

We have investigated the patterns of collision outcomes for a water droplet upon a surface covered by a layer with finite thickness of the same liquid. By changing the thickness, a regime diagram based on the variation of We was obtained, simulating a dry surface, a film, and a pool. The critical Weber numbers of transition boundaries between the regimes that were characterized by ejection of a central jet and that followed by a secondary droplet were found to decrease monotonically as $H$ was increased from a thin-film limit with $H$ being on the order of 0.1 , and approach a constant when $H$ was larger than 2 . The boundary designating occurrence of multiple droplets pinched from the central jet, however, is non-monotonic owing to a tuning mechanism, showing a minimum $W e$ at $H \approx 3$. These regimes are intercepted by a curve that indicates splattering of secondary droplets from the fingered rim of the sheet, leading to a splashing phenomenon. This boundary is invariant when $H$ is sufficiently high while the transitional We increases with decreased $H$ in the range of $1 \lesssim H \lesssim 2$; it is almost unchanged as $H \lesssim 1$. At even higher Weber number, a specific transition that indicated closure of the ejected sheet at the rim was found to locate around $W e=4,000$ in a thin- 
Fig. 12 The collision sequence of a droplet with $W e=2,621$ $(V=16.302 \mathrm{~m} / \mathrm{s}$,

$D=0.71 \mathrm{~mm}$ ) for $\mathbf{a} H=8.451$

$(\Delta t=2.34 \mathrm{~ms})$ and

b $H=0.845(\Delta t=1.17 \mathrm{~ms})$
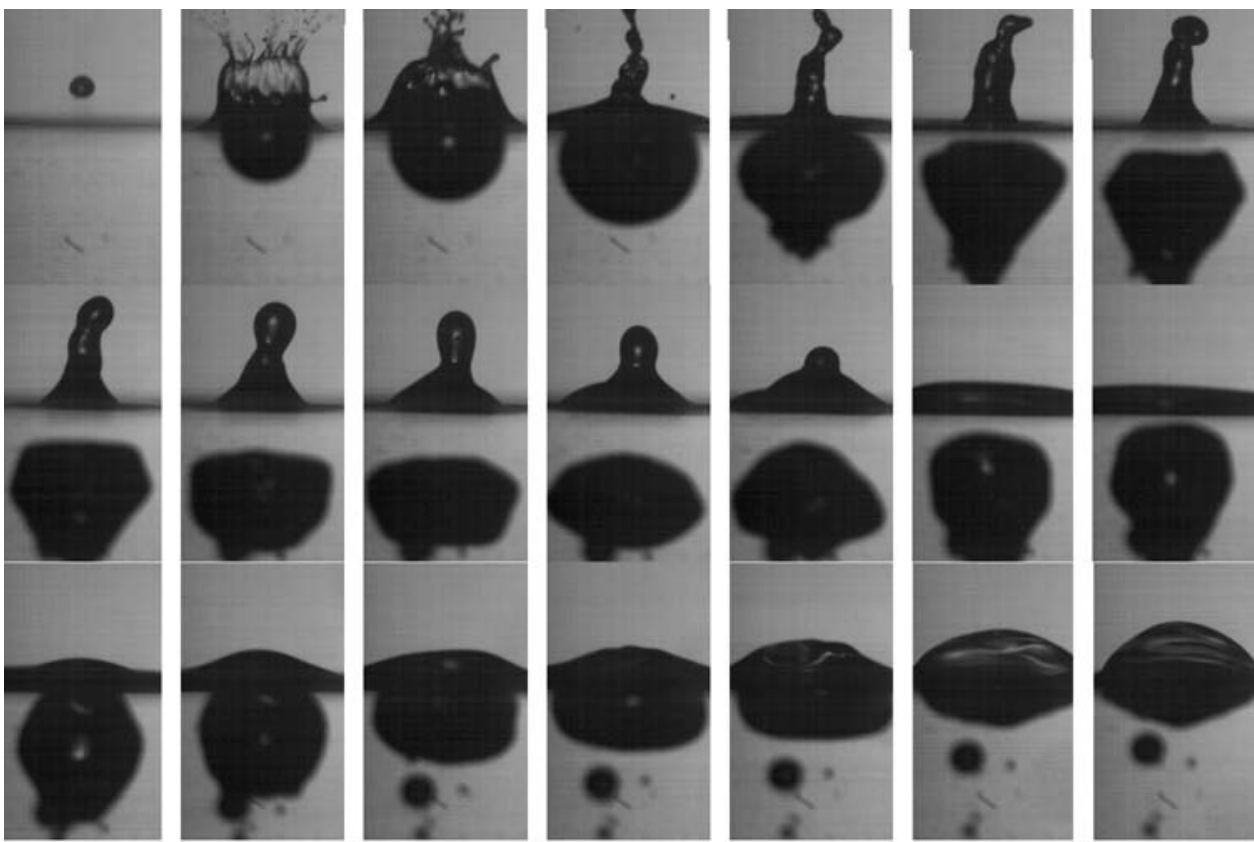

(a)
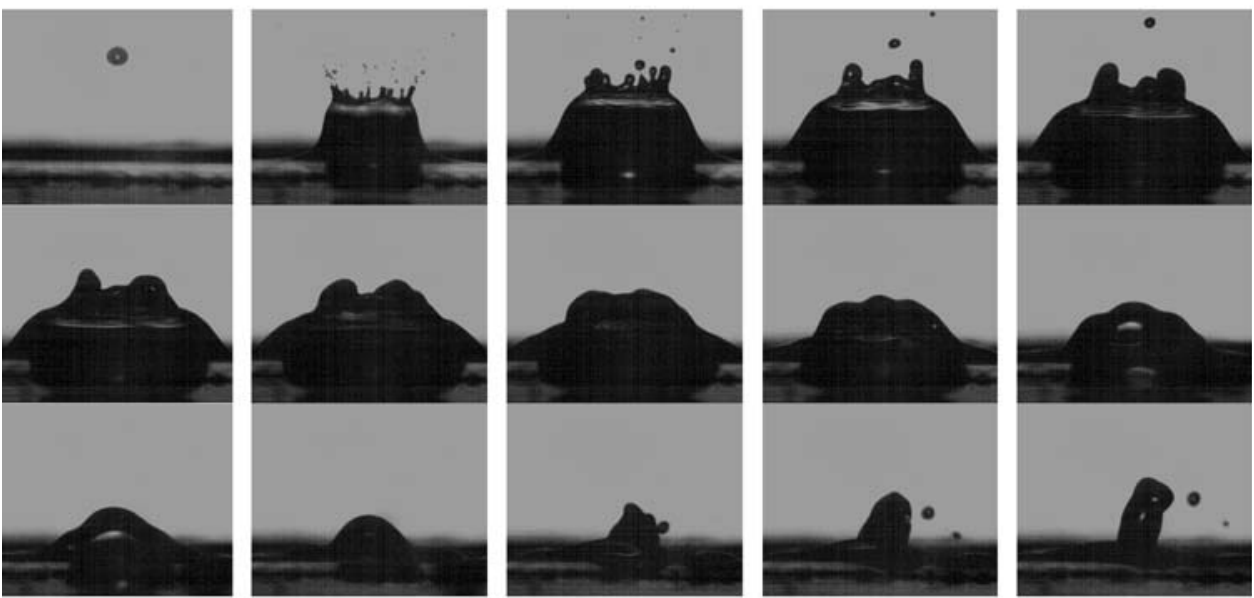

(b) film condition while it decreased to a constant of about 2,570 when the film thickness was larger than the droplet diameter. No other significant transition has been found as $W e$ is further increased up to 5,100, which is the limit of the present experiment.

Acknowledgments We thank National Science Council for the support (project number: NSC 94-2212-E-002-081 and 95-2221-E002-338).

\section{References}

Adam JR, Lindblad NR, Hendricks CD (1968) The collision, coalescence, and disruption of water droplets. J Appl Phys 39:5173-5180

Ashgriz N, Poo JY (1990) Coalescence and separation in binary collisions of liquid drops. J Fluid Mech 221:183-204
Bagué A, Zaleski S, Josserand C (2007) Droplet formation at the edge of a liquid sheet. In: M. Sommerfeld (ed) Proceedings of 6th international conference on multiphase flow, ICMF 2007, Leipzig

Chandra S, Avedisian CT (1991) On the collision of a droplet with a solid surface. Proc R Soc Lond A 432:13-41

Cossali GE, Coghe A, Marengo M (1997) The impact of a single drop on a wetted solid surface. Exp Fluids 22:463-472

Cossali GE, Marengo M, Coghe A, Zhdanov S (2004) The role of time in single drop splash on thin film. Exp Fluids 36:888-900

Cossali GE, Marengo M, Santini S (2005) Single-drop empirical models for spray impact on solid walls: a review. Atom Sprays 15:699-736

Couder Y, Fort E, Gautier CH, Boudaoud A (2005) From bouncing to floating: noncoalescence of drops on a fluid bath. Phys Rev Lett 94 (177801):1-4

de Gennes PG (1985) Wetting: statistics and dynamics. Rev Mod Phys 57:827-863

Eggers J (1997) Nonlinear dynamics and breakup of free-surface flows. Rev Mod Phys 69:865-929 
Engel OG (1966) Crater depth in fluid impacts. J Appl Phys 37:17981808

Hobbs PV, Kezweeny AJ (1967) Splashing of a water drop. Science 155:1112-1114

Hobbs PV, Osheroff TO (1967) Splashing of drops on shallow liquids. Science 158:1184-1186

Jayaratne OW, Mason BJ (1964) The coalescence and bouncing of water drops at an air/water interface. Proc R Soc Lond A 280:545-565

Jiang YJ, Umemura A, Law CK (1992) An experimental investigation on the collision behaviour of hydrocarbon droplets. J Fluid Mech 234:171-190

Macklin WC, Hobbs PV (1969) Subsurface phenomena and the splashing of drops on shallow liquids. Science 166:107-108

Macklin WC, Metaxas GJ (1976) Splashing of drops on liquid layers. J Appl Phys 47:3963-3970

Marmottant P, Villermaux E (2004) Fragmentation of stretched liquid ligaments. Phys Fluids 16:2732-2741

Okawa T, Shiraishi T, Mori T (2006) Production of secondary drops during the single water drop impact onto a plane water surface. Exp Fluids 41:965-974

Orme M, Muntz EP (1987) New technique for producing highly uniform droplets streams over an extended range of disturbance wavenumbers. Rev Sci Instrum 58:279-284

Pan KL, Law CK (2004) On the dynamics of head-on droplet collision: experiment and simulation. 42nd AIAA aerospace sciences meeting and exhibit, Reno, AIAA Paper No. 1159

Pan KL, Law CK (2007) Dynamics of droplet-film collision. J Fluid Mech 587:1-22

Qian J, Law CK (1997) Regimes of coalescence and separation in droplet collision. J Fluid Mech 331:59-80

Rayleigh L (1879) On the instability of jets. Proc Lond Math Soc 10:4-29

Rein M (1993) Phenomena of liquid drop impact on solid and liquid surfaces. Fluid Dyn Res 12:61-93
Rioboo R, Bauthier C, Conti J, Voué M, De Coninck J (2003) Experimental investigation of splash and crown formation during single drop impact on wetted surfaces. Exp Fluids 35:648-652

Rodriguez F, Mesler R (1985) Some drops don't splash. J Colloid Interface Sci 106:347-352

Roisman IV, Tropea C (2002) Impact of a drop onto a wetted wall: description of crown formation and propagation. J Fluid Mech 472:373-397

Roisman IV, Horvat K, Tropea C (2006) Spray impact: rim transverse instability initiating fingering and splash, and description of a secondary spray. Phys Fluids 18(102104):1-19

Shin J, McMahon TA (1990) The tuning of a splash. Phys Fluids A 2:1312-1317

Stow CD, Hadfield MG (1981) An experimental investigation of fluid flow resulting from the impact of a water drop with an unyielding dry surface. Proc R Soc Lond A 373:419-441

Thoroddsen ST (2002) The ejecta sheet generated by the impact of a drop. J Fluid Mech 451:376-381

Vander Wal RL, Berger GM, Mozes SD (2006) Droplets splashing upon films of the same fluid of various depths. Exp Fluids 40:3352

Wang AB, Chen CC (2000) Splashing impact of single drop onto very thin films. Phys Fluids 12:2155-2158

Worthington AM (1908) A study of splashes. Longmans, Green/ London

Xu L, Zhang WW, Nagel SR (2005) Drop splashing on a dry smooth surface. Phys Rev Lett 94 (184505):1-4

Yarin AL (2006) Drop impact dynamics: splashing, spreading, receding, bouncing. Annu Rev Fluid Mech 38:159-192

Yarin AL, Weiss DA (1995) Impact of drops on solid surfaces: selfsimilar capillary waves, and splashing as a new type of kinematic discontinuity. J Fluid Mech 283:141-173 